\title{
Block Reflectors: Theory and Computation
}

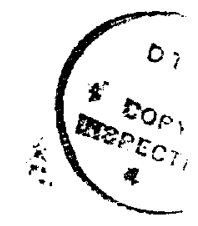

\author{
Robert Schreiber \\ Department of Computer Srience \\ Rensselaer Polytechnic Institute \\ Troy, New York 12180-3590

\section{and}

Beresford Parlett

Department of Mathematics

University of California Berkeley, Californiz 94720
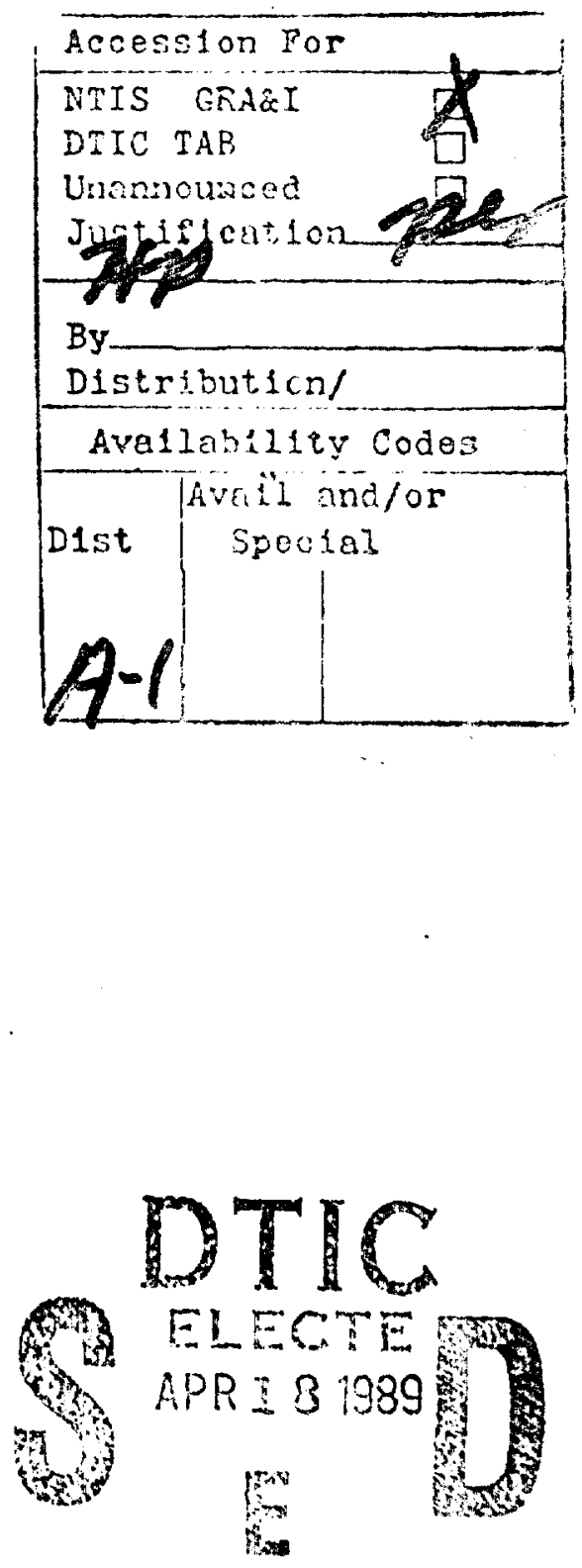

87-11

March, 1987

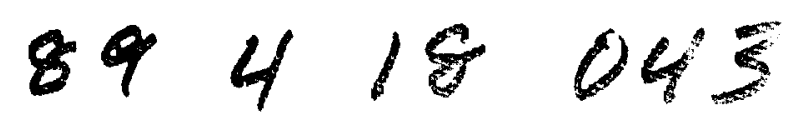





\section{BLOCK REFLECTORS: THEORY AND COMPUTATION"}

\section{ROBERT SCHREIBER $†$ AND BERESFORD PARLETT $\ddagger$}

Abstract. A block reflector is an orthogonal, symmetric matrix that reverses a subspace whose dimension may be greater than one. We shall develop the properties of block reflectors and give some algorithms for computing a block reflector that introduces a block of zeros into a matrix. We consider the compact. representation of block refiectors, some applications, and their use in parallel computers.

1. Introduction. Block reflectors are orthogonal, symmetric matrices with possibly more than one negative eigenvalue. They are a natural generalization of the clementary reflectors (also known as Houscholder transformations) that are widely used in matrix computation. Block reflectors have similar uses.

We shall develop a theory of block reflectors and their computation. We also discuss some applications of block reflectors, give some numerical results showing the stability of our algorithm, and show how this algorithm is well matched to the capabilities of some new, fast scientific computers.

Block reflectors are not new. Bronlund and Johnsen gave a method for orthogonal reduction to block upper triangular form, but the orthogonal transformations were nonsymmetric [2]. Dietrich derived the block reflector as we discuss it here, gave a stable method for computing it, and showed how it may be used for reduction to block upper triangular form [4]. Kaufman has considered the use of block reflectors for block triangularization of a sparse matrix [8].

In this paper, we make the following contributions. First, we derive a complete theory of block reflectors, clearly showing the parallels between the block and the point theory. Our presentation is considerably more direct than that found in [4].

† Department of Computer Science, Reneedser Polytechnic Institute, Troy, New York 12180 3590. This rewareb wa partially aupportad by the Sexpy Computer Corporation and the Office of Navel Research under Contract NoOO14-86-K-0010.

I Department of Mathematica, Univensity of California, Berkeley, California, 94720. This resench we partially supported by the Office of Naral Reeearch onder Contract NO0014-85-K-0180.

- SUAM Joural on Numerical Anabyais, to appear. A preliminary version of thit paper appeared in R. Glowinaki and J.L. Lions ods., Computer Methodo in Applied Sciences and Engincerine VII, Nortb-Holland, Amotardam, 1986. 
In addition to existence, we also answer the question of the uniqüeness of the block reflector, giving it in its most general form. Then we present a new view of the theory; our vantage point is the operator angle between two subspaces. This more clearly reveals the structure of the block reflector used to map between two subspaces, and also leads to new algorithms.

After providing two applications, we show how to construct a block reflector that introduces a zero block into a matrix. Four algorithms are presented. One is a new version of Dietrich's stable method. The role played by the polas decomposition of a matrix in this method is revealed. A second, related algorithm can be used to construct any of the several block reflectors that map between a given pair of subspares; Dietrich's method can be used to construct only one of these. Two new algorithms based on the operator angle are also given. Finally, the efficiency of these methods on modern parallel computers is examined. A numerical experiment illustrates their accuracy, even for very badly conditioned matrices.

Bischof and Van Loan have pursued a somewhat different approach [1]. They develop a representation for the product of several elementary reflectors of the form $I-W Y$, where $W$ and $Y$ are rectangular matrices. With this representation, the usual orthogonal reduction to triangular form can be organized so that it is dominated by matrix multiplications, an important virtue, as we explain below.

For any matrix $X, R(X)$ denotes the range of $X$. For any subspace $Y, Y^{\perp}$ denotes the orthogonal complement of $Y$.

2. Block reflectors: Theory.

3.1. Definition. Given any $Z \in \mathbf{R}^{m \times n}, m \geq n$, the refiector that reverses the range of $Z^{n}$ is given by

$$
H=H(Z):=I_{m}-Z W Z^{l}
$$

where

$$
W=2\left(Z^{t} Z\right)^{+} \in \mathbf{R}^{n \times n}
$$

is the (symmetric) pseudo-inverse of $\frac{1}{2}\left(Z^{t} Z\right)$. (See [6, p. 139] for a fuller description of the pseudo-inverse.) Thus

$$
Z W Z^{t}=2 P_{Z}
$$

where $P_{Z}$ is the orthogonal projector on $R(Z)$.

When $n=1, H$ is an elementary reflector or Householder transformation. When $Z^{t} Z$ is invertibie, $W=\left(\frac{1}{2} Z^{t} Z\right)^{-1}$. Note that if $Z=0$, then $W=0$ and $\boldsymbol{H}(0)=I$. Aleo, $H(Z) Z=-Z$. (See Lemma 1 for proof.)

This choice of $W$ makes $H$ orthogonal as well as symmetric. Hence, $H^{2}=I$, the refiector property.

It is also easy to verify that if $R(Z)=R\left(Z_{1}\right)$, then $H(Z)=B\left(Z_{1}\right)$. Thus, for example, $H(Z T)=H(Z)$ for any invertible $T$, and $H(Z W)=H(Z)$ as well. 
Since $H$ is orthogonal and symmetric, its eigenvalues are 1 and -1 . The multiplicity of -1 is equal to the dimension of the space reversed by $H$; this in turn is equal to the rank of $Z$.

2.2. Essential properties. Every $m \times m$ orthogonal reflector $H$ induces a decomposition of $\mathbf{R}^{m}$ into the direct sum of two perpendicular subspaces; one subspace is $H$ invariant and the other is reversed by $H$. The following lemma states that for the reflector $H(Z)$, the reversed subspace is $R(Z)$.

LEMMA 1. Let $Z \in \mathbf{R}^{m \times n}$. For all $z \in R(Z)$,

$$
H(Z) z=-z
$$

and for all $y \in R(Z)^{\perp}$,

$$
H(Z) y=y
$$

Proof. We require the fact that, for any matrix $B$,

$$
B\left(B^{t} B\right)^{+} B^{t} B=B \text {, }
$$

which is easy to prove using the singular value decomposition (SVD) of $B$. (See $\mid 6$, pp. 16-20! for more information on the SVD.) Now let $z \in R(Z)$. Then there exists $x$, such that $z=Z x$. Using the fact above and the definition of $H(Z)$,

$$
\begin{aligned}
H(Z) z & =Z x-Z W Z^{l} Z_{x} \\
& =Z_{x}-2 Z x \\
& =-z
\end{aligned}
$$

so that (1a) is proved. Property (1b) comes from applying $H(Z)$ to $y$ and noting that $Z^{t} y=0$.

QED

2.3. The standard task. Let $E=\left(\begin{array}{l}E_{1} \\ E_{2}\end{array}\right) \in \mathrm{R}^{m \times n}$, with $m>n$, and $E_{1}$ square. We seek a block reflector $H$ such that

$$
H E=\bar{F}:=\left(\begin{array}{l}
F \\
0
\end{array}\right)
$$

with $F$ square. We now give conditions on $F$ that are necessary and sufficient for the existence of $H$ :

$$
\text { ISOMETRY PROPERTY: } F^{t} F=E^{t} H^{t} H E=E^{\ell} E
$$

and

$$
\text { SYMMETRY PROPERTY: } E_{1}^{\ell} F=E^{t} H E=\text { symmetric. }
$$


Define the associated matrices:

(3a)

$$
\begin{aligned}
D: & =\bar{F}-E \\
& =\left[\begin{array}{c}
F-E_{1} \\
-E_{2}
\end{array}\right]
\end{aligned}
$$

and

$$
S:=\bar{F}+E \text {. }
$$

( $D$ is for "difference," $S$ is for "sum.")

An important consequence of the conditions (2) is this:

LEMMA 2. If $F$ satisfies (2), then

$$
D^{t} S=0 .
$$

Proof. By (2),

$$
\begin{aligned}
D^{t} S & =\left(\bar{F}^{t} \bar{F}-E^{t} E\right)+\left(\bar{F}^{t} E-E^{t} \bar{F}\right) \\
& =\left(F^{t} F-E^{t} E\right)+\left(F^{t} E_{1}-E_{1}^{t} F\right) \\
& =0+0 .
\end{aligned}
$$

THFORFM 1. There exists a block reflector $H$ such that $H E=\bar{F}$ if and only if $F$ satisfies (2).

Proof. Necessity is clear: equation (2a) follows from the orthogonality of $H$, while $(2 \mathrm{~b})$ is obvious from the symmetry of $H$. have

To show sufficiency we shall prove that $H(D) E=F$. Since $E=\frac{1}{2}(S-D)$, we

$$
\begin{aligned}
H(D) E & =\frac{1}{2}[H(D) S-H(D) D] \\
& =\frac{1}{2}[S+D] \quad \text { (using Lemmas } 1 \text { and 2) } \\
& =\frac{F}{}
\end{aligned}
$$

Clearly if $F$ satisfies (2) then $-F$ does too. Furthermore, $H(S) E=-\bar{F}$. If $F$ and $E$ satiofy (2) we shall say that $F$ is a mirror image of $E$.

The concept of a block reflector, the necessary and sufficient conditions, and a solution to the standard task for the case $n=2$ were given by Tang Ling in an unpublished manuscript [10].

2.3.1. Representing $H$. It may not be advisable to represent $H$ by $E, F$, and $W$ as above. This form is very attractive if $E$ must be preserved or when $E$ is 
sparse. On the other hand, when $E$ has rank $r<n$. then it saves storage to find an $m \times r$ matrix $G$ such that $H=I-G G^{t}$, and $G^{t} G \cdot 2 I_{r}$. (We later show that this is possible). Storage of $G$ requires $m r$ words, as opposed to $m n+n^{2}$ for $Z$ and $W, m n+2 n^{2}$ for $E, F$, and $W$, and $2 m n$ for the $W Y$ representation of Bischof and Van Loan. Computing $H x$ for a vector $x$ costs $2 m r$ flops ( 1 flop is one multiply and one add) compared with $2 m n+n^{2}$ for the $Z, W$ representation. An algorithm for computing $\mathbf{G}$ is given in $\$ 3.2$.

2.4. The form of $F, F$ is far from unique, even when $E$ has full rank $n$. If $E_{1}=0$ then the symmetry condition is vacuous and we may choose any $F$ satisfying the isometry condition $F^{t} F=E^{t} E$. At the other extreme, when $E_{1}$ is invertible, then $F$ must have the form $F=M E_{1}$ with $M$ symmetric. Now the isometry condition requires

$$
M^{2}-E_{1}^{-t}\left(E^{\prime} E\right) E_{1}^{-1}
$$

There are $2^{n}$ solutions, namely $M=V \operatorname{diag}\left( \pm \sqrt{\lambda_{1}}, \pm \sqrt{\lambda_{2}}, \cdots, \pm \sqrt{\lambda_{n}}\right) V^{t}$ where

$$
E_{1}^{-t}\left(E^{t} E\right) E_{1}^{-1}=V \operatorname{diag}\left(\lambda_{1}, \cdots, \lambda_{n}\right) V^{t}
$$

is the spectral factorization. Note that there are $2^{n}$ different solutions for every spectral factorization of $E^{-t}\left(E^{t} E\right) E^{-1}$. And with repeated eigenvalues there are infinitely many such factorizations.

The two "extreme" solutions are $F= \pm\left[E_{1}^{-t}\left(E^{t} E\right) E_{1}^{-1}\right]^{1 / 2} E_{1}$, where $A^{1 / 2}$ is the positive definite square root of $A$.

Example. Let

$$
E=\left[\begin{array}{l}
V \\
0
\end{array}\right]
$$

where $V^{t}=V^{-1}$; i.e., $V$ is orthogonal. Then $E_{1}^{-1}\left(E^{t} E\right) E_{1}^{-1}=V V^{t}=I$. Thus we may take $M$ to be any symmetric orthogonal matrix $\left(M^{2}=I\right)$; in particular, $M=I$ will do. Then $F=M V$, and

$$
H E=\left(\begin{array}{cc}
M & 0 \\
0 & I
\end{array}\right)\left(\begin{array}{l}
V \\
0
\end{array}\right)=\left(\begin{array}{l}
F \\
0
\end{array}\right)
$$

2.4.1. The general case. Let $E_{1}=P \Sigma Q^{t}$ with $P^{t} P=Q^{t} Q=I_{r_{1}}, \Sigma$ positive definite and diagonal, $r_{1} \leq n$. This is the "short" SVD of $E_{1}$, where $r_{1}=\operatorname{rank}\left(E_{1}\right)$. The symmetry condition $(2 b)$ requires, that

$$
Q \Sigma P F=\text { symmetric. }
$$

The general solution for $F$ is

$$
F=(P, \tilde{P})\left(\begin{array}{cc}
M_{11} & 0 \\
M_{21} & M_{22}
\end{array}\right)\left(\begin{array}{cc}
\Sigma & 0 \\
0 & I_{n-r_{1}}
\end{array}\right)(Q, \bar{Q})^{t}
$$


where $\tilde{P}, \tilde{Q} \in \mathbf{R}^{n \times\left(n-r_{1}\right)}$ make $(P, \tilde{P})$ and $(Q, \tilde{Q})$ orthogonal. $M_{11}$ must be symmetric, but $M_{21}$ and $M_{22}$ are free.

The isometry condition (2a) yields

$$
\left(\begin{array}{cc}
M_{11} & M_{21}^{t} \\
0 & M_{22}^{t}
\end{array}\right)\left(\begin{array}{ll}
M_{11} & 0 \\
M_{21} & M_{22}
\end{array}\right)=\left(\begin{array}{cc}
\Sigma^{-1} & 0 \\
0 & I
\end{array}\right)(Q, \dot{Q})^{t} E^{t} E(Q, \tilde{Q})\left(\begin{array}{cc}
\Sigma^{-1} & 0 \\
0 & I
\end{array}\right)
$$

For each choice of $\dot{P}$ and $\dot{Q}$ we can find $M_{11}, M_{21}$, and $M_{22}$ such that this equation holds. The solutions are not unique. We solve

$$
\begin{aligned}
M_{22}^{t} M_{22} & =\dot{Q}^{t} E^{t} E \bar{Q} \\
M_{22}^{t} M_{21} & =\dot{Q}^{t} E^{t} E Q \Sigma^{-1} \\
M_{11}^{2} & =\Sigma^{-1}(E Q)^{t}(E Q) \Sigma^{-1}-M_{21}^{\ell} M_{21}
\end{aligned}
$$

Even when $M_{22}$ is singular, (4b) is consistent. Nevertheless, the system (4b) may be ill conditioned, so we do not propose to use (4) in computations.

\subsection{Is $H$ unique?}

Suppose $F$ satisfies the isometry and symmetry conditions (2). We have shown that the choice $Z=D$ provides a block reflector $H$ such that $H E=\bar{F}$. Is this the only such $H(Z)$ ? The answer depends on the rank of $D$ and the rank of $S$ in (3) above.

THEOREM 2. Let $F$ satisfy (2). Let $H=H(Z)$. The conditions

$$
R(D) \subseteq R(Z)
$$

and

$$
R(Z) \perp R(S), \quad \text { i.e., } \quad Z^{\ell} S=0
$$

are necessary and sufficient for $H(Z) E$ to be equal to $\bar{F}$.

Proof. Recall that $E=\frac{1}{2}(S-D)$. If $Z$ satisfies (5) then, as in the proof of Theorem 1,

$$
\begin{aligned}
H(Z) E & =\frac{1}{2}(H S-H D) \\
& =\frac{1}{2}(S+D) \\
& =\frac{F}{F}
\end{aligned}
$$

by Lemmas 1 and 2.

On the other hand, if $H E=\bar{F}$, then

$$
F=E-Z W Z^{t} E,
$$


so that

$$
\begin{aligned}
D & =-Z W^{\prime} Z^{t} E \\
& =Z\left(\cdot W Z^{\ell} E\right)
\end{aligned}
$$

which implies (5a). Now that (5a) is established. we may use it to prove that (5b) holds. A consequence of $(5 a)$ is that $H(Z) D=-D$. Rewriting this relation yields

$$
Z W Z^{t} D=2 D
$$

Using this equation we obtain

$$
\begin{aligned}
\bar{F} & =H E \\
& =E-\frac{1}{2} Z W Z^{t}(S-D) \\
& =E+D-\frac{1}{2} Z W Z^{t} S \\
& =\bar{F}-\frac{1}{2} Z W Z^{t} S .
\end{aligned}
$$

So $Z W Z^{t} S=0$. Since, as we noted earlier, $\frac{1}{2} Z W Z^{t}$ is the orthogonal projector on $\boldsymbol{R}(Z)$, the columns of $S$ are orthogonal to those of $Z$. This implies (5b). QED

Now suppose that $H(Z) E=\bar{F}$. By Theorem 1, $F$ satisfies (2) and by Theorem $2, Z$ satisfies (5). Let us define

$$
\rho Z=\operatorname{rank}(Z), \quad \rho D=\operatorname{rank}(D), \quad \rho S=\operatorname{rank}(S)
$$

Since $Z, D$, and $S$ belong to $\mathbf{R}^{\mathrm{m} \times \boldsymbol{\pi}}$,

$$
\rho 2 \leq n ; \quad \rho D \leq n ; \quad \rho S \leq n .
$$

- Further, by (5a)

$$
\rho D \leq \rho z
$$

and by (5b)

$$
\rho_{z} \leq m-\rho s
$$

Since $F$ satisfies (2), Lemma 2 applies, so that

$$
\rho_{D}+\rho S \leq m
$$

(We could conclude (6d) from (6b) and (6c), but it is true independent of the existence of $Z$, as we have shown.)

Now we can say when $H$ is unique. 
THEOREM 3. Let $F$ be a mirror image of $E$. Then

1. if $\rho_{D}=n$, then $H(D)$ is the unique bluck reflector satisfying $H E=\bar{F}$;

8. if $\rho D+\rho S=m$, then $H(D)$ is the unique block reflector satisfying $H E=\bar{F}$;

9. if $\rho D<\min (n, m-\rho S)$, then $H$ is not unique.

Proof. Suppose $H(Z) E=\bar{F}$. If $\rho D=n$, then it is clear from (5a) and (6a) that $R(Z)=R(D)$, so $H(Z)=H(D)$ is the unique block reflector that reverses $R(D)$. Similarly, if $\rho_{D}+\rho_{S}=m$, then by $(5 \mathrm{a}),(5 \mathrm{~b}),(6 \mathrm{~b})$ and $(6 \mathrm{c})$, we must have $R(Z)=R(D)$, and again $H(Z)=H(D)$ is the unique block reflector that reverses $R(D)$. Finally, if $\rho_{D}<\min \left(n, m-\rho_{S}\right)$, we may choose any matrix $Z \subseteq \mathbf{R}^{m \times n}$ whose range contains $R(D)$ and is orthogonal to $R(S)$. By our assumption, there exist such $Z$ of rank $\rho D, \rho_{D}+1, \ldots, \min (n, m-\rho S)$.

QED

COROLlARY. If $E_{2}$ has rank $n$, then $H$ is unique.

Proof. Since

$$
D=\left(\begin{array}{c}
F-E_{1} \\
-E_{2}
\end{array}\right)
$$

it follows that $n \geq \operatorname{rank}(D) \geq \operatorname{rank}\left(E_{2}\right)=n$.

In the case of Householder transformations $(n=1)$, the condition of the corot lary is satisfied unless $E_{2}=0$, in which case the standard task is not much of a task at all! Thus, like the symmetry condition (2b), the possibility of genuine nonuniqueness of the reflector $H$ only appears in the multidimensional case.

Examples. Let

$$
E=\left[\begin{array}{ll}
1 & 0 \\
0 & 1 \\
0 & 0
\end{array}\right]
$$

The three matrices

$$
F_{0}=\left(\begin{array}{ll}
1 & 0 \\
0 & 1
\end{array}\right), \quad F_{1}=\left(\begin{array}{cc}
1 & 0 \\
0 & -1
\end{array}\right), \quad F_{2}=\left(\begin{array}{cc}
-1 & 0 \\
0 & -1
\end{array}\right)
$$

all satisfy the conditions (2). With $F_{0}, \rho D=0$ and $\rho s=2$ and we have $0 \leq \rho_{Z} \leq 1$. We may choose

$$
H_{0}=\left[\begin{array}{ccc}
1 & 0 & 0 \\
0 & 1 & 0 \\
0 & 0 & \pm 1
\end{array}\right] .
$$

With $F_{1}$ we have $\rho D=1$ and $\rho_{S}=1$ and $1 \leq \rho z \leq 2$. We may choose

$$
H_{1}=\left[\begin{array}{ccc}
1 & 0 & 0 \\
0 & -1 & 0 \\
0 & 0 & \pm 1
\end{array}\right]
$$

With $F_{2}$ we have $\rho D=2$ and $\rho S=0$ and $2 \leq \rho Z \leq 2$. We must choose

$$
H_{2}=\left[\begin{array}{ccc}
-1 & 0 & 0 \\
0 & -1 & 0 \\
0 & 0 & 1
\end{array}\right]
$$


This example shows that nonuniqueness of $F$ is possible anc that uniqueness of $H$ is possible even with singular $E_{2}$.

For a contrasting example, let

$$
E=\left[\begin{array}{lll}
0 & 0 & 0 \\
0 & 0 & 0 \\
1 & 0 & 1 \\
0 & 1 & 1
\end{array}\right], \quad \bar{F}=\left[\begin{array}{lll}
1 & 0 & 1 \\
0 & 1 & 1 \\
0 & 0 & 0 \\
0 & 0 & 0
\end{array}\right] .
$$

Then $\rho_{D}=2, \rho_{S}=2$, al, $2 \leq \rho_{Z} \leq 2$. Thus $H=H(D)$ is unique, even though $E$ is rank-deficient.

2.6. The angle between $R(E)$ and $R\left(\left[\begin{array}{l}I \\ 0\end{array}\right]\right)$. In this section we shall rederive many of our results using the operator angle between $R(E)$ and $R\left(\left[\begin{array}{l}I \\ 0\end{array}\right]\right)$. For a complete discussion of the angle between subspaces see Davis and Kahan [3]. This rederivation gives us a new view of the block reflector that allows some geometric insight not available otherwise. It also leads to some algorithms that would not be discovered from the algebraic perspective of the earlier sections.

Let $r \equiv \operatorname{rank}(E)$. Let the columns of $P \in \mathbf{R}^{m \times r}$ be an orthonormal basis for $R(E)$. We discuss the problem of finding a block reflector $H$ that performs the standard task for $P$ rather than $E$; since they have the same range, this $H$ also performs the standard task for $E$ : If $E=P T$ and $H$ is a block reflector such that $H P=\left[\begin{array}{l}Q \\ 0\end{array}\right], Q$ square, then $H E=\left[\begin{array}{c}Q T \\ 0\end{array}\right]$. Thus, for the moment, we work with $P$ rather than $E$.

Let

$$
\boldsymbol{P}=\left[\begin{array}{l}
\boldsymbol{P}_{1} \\
\boldsymbol{P}_{2}
\end{array}\right]
$$

where $P_{1}$ is square. Let

$$
P_{1}=Q_{1} M_{1}
$$

be a polar decomposition of $P_{1}$. The factor $M_{1}$ is the symmetric, nonnegative definite square root of $P_{1}^{t} P_{1}$, and is unique. The other factor, $Q_{1}$, is orthogonal and is unique only if $P_{1}$ is nonsingular. Since $P$ has orthonormal columns, the eigenvalues of $M_{1}$ all lie in $[0,1]$ (see $[6$, p. 22]). Higham $[7]$ discusses the polar decomposition and gives an efficient algorithm for computing it.

It is simple to show that $Q_{1}$ is a mirror image of $P$. In fact, a version of the $\cdots$ converse is also true. If $Q$ is any mirror image of $P$ then

$$
P_{1}=Q M
$$

is a factorization of $P_{1}$ into an orthogonal-symmetric product. (The choice is in the signs of the eigenvalues of $M$, as it was in \$2.4). For the moment we choose to work with the polar factorization. 
Define $\overline{Q_{1}} \equiv\left(\begin{array}{c}Q_{1} \\ 0\end{array}\right)$. We now write

$$
M_{1}=\cos 2 \theta
$$

where the angle $\theta$ is defined by

$$
\theta \equiv V \operatorname{diag}\left(\theta_{1}, \cdots, \theta_{r}\right) V^{t}
$$

where

$$
0 \leq \theta_{1} \leq \cdots \leq \theta_{r} \leq \frac{\pi}{4} \text {. }
$$

The eigenvectors of $M_{1}$ are the columns of $r$ and its eigenvalues are $1 \geq \cos 2 \theta_{1} \geq$ $\cdots \geq \cos 2 \theta, \geq 0$.

We also factor

$$
P_{2}=Q_{2} M_{2}
$$

where $M_{2}=\left(I-M_{1}^{2}\right)^{1 / 2}=\sin 2 \theta$ is symmetric, nonnegative definite and $Q_{2} \in$ $\mathbf{R}^{m-r \times r}$. The form of $Q_{2}$ will be clarified below. We may choose $Q_{2}$ so that

$$
Q_{2}^{\ell} Q_{2}(\sin \theta)=\sin \theta
$$

It is easy to prove this by using the C-S decomposition of $P$ [13].

From this new viewpoint we obtain several new formulas. First

$$
P_{1}=Q_{1} M_{1}=Q_{1} \cos 2 \theta, \quad P_{2}=Q_{2} \dot{M}_{2}=Q_{2} \sin 2 \theta
$$

hence

$$
\left(I+M_{1}\right)^{1 / 2}=\sqrt{2} \cos \theta, \quad\left(I-M_{1}\right)^{1 / 2}=\sqrt{2} \sin \theta
$$

Next,

$$
S=\left(\begin{array}{c}
Q_{1}+P_{1} \\
P_{2}
\end{array}\right)=\left(\begin{array}{c}
2 Q_{1} \cos ^{2} \theta \\
P_{2}
\end{array}\right)=2\left(\begin{array}{c}
Q_{1} \cos \theta \\
Q_{2} \sin \theta
\end{array}\right) \cos \theta
$$

and

$$
D=\left(\begin{array}{c}
\mathscr{P}_{1}-P_{1} \\
-P_{2}
\end{array}\right)=\left(\begin{array}{c}
2 Q_{1} \sin ^{2} \theta \\
-P_{2}
\end{array}\right)=2\left(\begin{array}{c}
Q_{1} \sin \theta \\
-Q_{2} \cos \theta
\end{array}\right) \sin \theta
$$

Thus,

$$
\frac{1}{2} s^{t} S=2 \cos ^{2} \theta
$$


and

$$
\frac{1}{2} D^{t} D=2 \sin ^{2} \theta
$$

The definition of the angles $\left\{\theta_{j}\right\}$ insures that $\cos ^{2} \theta$ is both nonsingular and well conditioned. Define

$$
H_{+}=I-G_{+} G_{+}^{l}
$$

where

$$
G_{+} \equiv \sqrt{2}\left(\begin{array}{l}
Q_{1} \cos \theta \\
Q_{2} \sin \theta
\end{array}\right)
$$

By $(10)$ and $(12)$, since $\left(\cos ^{2} \theta\right)^{+}=\left(\cos ^{2} \theta\right)^{1}$,

$$
H(S)=H_{+} \text {. }
$$

Note the analogy with the case $n=1$ where, if $2 \theta$ is the angle between $e$ (that is, $E)$ and the $e_{1}$-axis, then $H=I \cdot 2 v_{+} v_{+}^{t}$, where $v_{+}=(\cos \theta, \sin \theta)^{t}$.

We now consider $H(D)$. It is important to be able to construct $H(D)$ since in some instances it is what we want. In particular, if $E \approx\left(\begin{array}{c}\text { orthogonal } \\ 0\end{array}\right)$ then $H(D)$ produces a small change to $\boldsymbol{E}$.

Now note that $D$ is not necressarily of full rank. In fact

$$
\begin{aligned}
P D & =: \operatorname{rank}(D) \\
& =\operatorname{rank}\left(D^{t} D\right) \\
& =\operatorname{rank}(\sin \theta) \\
& =\operatorname{rank}(\sin 2 \theta) \\
& =\operatorname{rank}\left(M_{2}\right) \\
& =\operatorname{rank}\left(P_{2}\right) \\
& \leq \min (r, m-r) \equiv s .
\end{aligned}
$$

If any of the $s$ singular values of $P_{2}$ is zero then $P D<s$ and the angles $\theta_{1}=\cdots=$ $\theta_{r-P D}=0$. In this case the analog to (14), namely

$$
H_{-}=I-G_{-} G_{-}^{t}
$$

where

$$
G_{-}=\sqrt{2}\left(\begin{array}{c}
Q_{1} \sin \theta \\
-Q_{2} \cos \theta
\end{array}\right)
$$

fails in the sense that $\boldsymbol{H}(D) \neq H_{-}$. For it is clear that $\operatorname{rank}\left(G_{-}\right)=8$, so that $H_{-}$ reverses an e-dimensional subspace; but $H(D)$ reverses only $R(D)$, which is just OD-dimensional. Since, by asumption, $\rho D<\bullet$, the two block refectors must difer. 
By Theorem 3, however, $H(D)$ is not unique. We may therefore ask whether $H_{\text {- satisfies }} H_{-} P=\overline{Q_{1}}$ despite the fact that it is not $H(D)$. According to Theorem 2 , it does if

$$
R(D) \subseteq R\left(G_{-}\right)
$$

and

$$
G_{-}^{l} S=0
$$

But (18) follows from the characterization (11) of $D$ and the definition (17) of $G_{-}$. The orthogonality (19) follows from property (8) of $Q_{2}$.

When $D$ is rank deficient there may be other choices as well. In fact, if $P D<$ $s-1$ then there are block reffectors that reflect $P$ into $\overline{Q_{1}}$ and reverse subspaces of dimension greater than $P D$ and less than $s$. The two that we have exhibited are the extreme cases: $H(D)$ reverses the smallest possible subspace $|R(D)|$, while $H_{-}$ reverses the largest $\left[R(S)^{\perp} \mid\right.$.

2.6.1. Other choices for $F$. Were we to take any other orthogonalsymmetric factorization $\left(Q_{1} M_{1}\right.$ with $M_{1}$ indefinite) of $P_{1}$, we could still construct block reflectors $H_{ \pm}$by (14) and (16). Now, in general, neither $H(S)=H_{+}$nor $H(D)=H_{\text {. }}$. But nevertheless, $H_{ \pm} P=\mp \overline{Q_{1}}$.

2.6.2. Other representations for $H$. When $m=2 r$ we may write

$$
H_{ \pm}=\mp\left(Q_{1} \oplus Q_{2}\right)\left[\begin{array}{cc}
\cos 2 \theta & \sin 2 \theta \\
\sin 2 \theta & -\cos 2 \theta
\end{array}\right]\left(Q_{1} \oplus Q_{2}\right)^{t}
$$

in exact analogy to the elementary case. In general, $m \neq 2 r$ and we have that

$$
H_{ \pm}=\left(\begin{array}{ll}
0 & 0 \\
0 & I-Q_{2} Q_{2}^{t}
\end{array}\right) \mp\left(Q_{1} \oplus Q_{2}\right)\left[\begin{array}{cc}
\cos 2 \theta & \sin 2 \theta \\
\sin 2 \theta & -\cos 2 \theta
\end{array}\right]\left(Q_{1} \oplus Q_{2}\right)^{t}
$$

\section{Applications and computation.}

\subsection{Applications.}

1. Optimal error bounds. Let the columns of $U$ be approximate eigenvectors for some symmetric $A \in \mathbf{R}^{m \times m}$. Let $\Lambda=\operatorname{diag}\left(\theta_{1}, \cdots, \theta_{n}\right)$ be approximate eigenvalues. Let $X:=A U-U A$ be a residual matrix. Next map $X$ into its mirror image $\left(\begin{array}{l}Y \\ 0\end{array}\right)$ by a suitable block reflector $H$. Then form the auxiliary symmetric matrix

$$
T=T(V)=\left(\begin{array}{ll}
\Lambda & Y^{\ell} \\
Y & V
\end{array}\right)
$$

where $V$ is at our disposal.

By choosing suitable $V$ and computing the eigenvalues of $T(V)$ error bounds may be obtained on the approximate values $\theta_{1}, \cdots, U_{n}$. In ceveral important cases 
$V$ can be chosen so that the bounds are optimal for the given information. See [11, $\$ \$ 10-4-10-9 \mid$ for more details.

The point of interest here is that the residual matrix $X$ is likely to have lower rank than is revealed by its columns alone.

2. Block Hessenberg form. It is possible to reduce a matrix $B \in \mathbf{R}^{m \times m}$ to block upper Hessenberg form by explicit orthogonal similarity transformations

$$
B \rightarrow C=H^{t} B H=\left[\begin{array}{ccccc}
C_{11} & C_{12} & C_{13} & C_{14} & C_{15} \\
C_{21} & C_{22} & C_{23} & C_{24} & C_{25} \\
0 & C_{32} & C_{33} & C_{34} & C_{35} \\
0 & 0 & C_{43} & C_{44} & C_{45} \\
0 & 0 & 0 & C_{54} & C_{55}
\end{array}\right]
$$

Here $\mathrm{H}$ represents a product of three block reflectors, $\mathrm{H}=\mathrm{H}_{1} \mathrm{H}_{2} \mathrm{H}_{3}$. The first step is typical. We seek $H_{1}$ so that

$$
H_{1}\left[\begin{array}{l}
B_{21} \\
B_{31} \\
B_{41} \\
B_{51}
\end{array}\right]=\left[\begin{array}{c}
C_{21} \\
0 \\
0 \\
0
\end{array}\right]
$$

In tnese circumstances we expect full rank to be maintained. It may not pay to try and represent $H_{1}=I_{m}-G_{1} G_{1}^{t}$ where $G_{1} \in R^{m \times r}$ since usually $r=n$.

Block $Q R$ factorizations can be computed in a similar manner, by applying a sequence of block reflectors to a matrix $[2,4]$.

3.2. Stable computation of the block reflector. Recall that $E \in \mathbf{R}^{m \times n}$ is given and we seek a block reflector $H=H(Z)$ such that $H E=\bar{F}=\left(\begin{array}{l}F \\ 0\end{array}\right)$ for some $n \times n$ matrix $F$. In this section we shall describe four elegant and stable constructions for mirror images $F$ of $E$ and of matrices $G \in \mathbf{R}^{m \times r}$ such that $G^{t} G=2 I$, and the block reflector $H=I-G G^{t}$ maps between $R(E)$ and $R\left(\left[\begin{array}{l}I \\ 0\end{array}\right]\right)$. One of these, Algorithm 2, appears in a slightly different form in [4].

As in \$2.6, we suppose we have a matrix $P \in \mathbf{R}^{m \times P}$ such that $R(E) \subset R(P)$ and $P$ has orthonormal columns. Thus $P^{t} P=I_{r}$, and $E=P T$ for some $T \in \mathbf{R}^{r \times n}$. We can easily find $T$ since

$$
\boldsymbol{T}=\boldsymbol{P}^{t} \boldsymbol{E}
$$

Let $P=\left[\begin{array}{l}P_{1} \\ P_{2}\end{array}\right]$ with $P_{1}$ square. Let $P_{1}=Q_{1} M_{1}$ be a polar decomposition of $P_{1}$. The orthogonal polar factor $Q_{1}$ is a mirror image of $P$. With this choice,

$$
s=\left[\begin{array}{c}
Q_{1}+P_{1} \\
P_{2}
\end{array}\right]
$$

Then as we have seen, $B=B(S)$ satisfies $H P=\left[\begin{array}{c}-Q_{1} \\ 0\end{array}\right]$, which is our objective. 
It remains only to find a convenient representation for $H$. The one given by (14)-(15) is a possibility. We shall compute the necessary matrices $\cos \theta$ and $\sin \theta$ using (9). In the computation of $\sin \theta$, however, it is possible that cancellation of nearly equal elements on the diagonal can spoil the formation of $I-M_{1}$. Following $[11$, p. 91$]$ in the one-dimensional case, we may use the relation

$$
\begin{aligned}
I-M_{1} & =\left(I+M_{1}\right)^{-1}\left(I-M_{1}^{2}\right) \\
& =\left(I+M_{1}\right)^{-1}\left(I-P_{1}^{t} P_{1}\right) \\
& =\left(I+M_{1}\right)^{-1}\left(P_{2}^{t} P_{2}\right)
\end{aligned}
$$

to construct $I-M_{1}$ without any matrix subtraction.

This leads to the following algorithm. Rerall that $M_{1}=\cos 2 \theta$ and $M_{2}=$ $\sin 2 \theta$.

AlgoRithM 1. Compute $F \in \mathbf{R}^{n \times n}$ and $G_{+} \div \mathbf{R}^{m \times r}, r \leq n$, such that $H_{+} E=\bar{F}$, where $H_{+} \equiv I-G_{+} G_{+}^{t}$.

1. Find $P \in \mathbf{R}^{m \times r}$ and $T \in \mathbf{R}^{r \times n}$ such that $R(E) \subseteq R(P), P^{t} P=I$, and $P T=E$;

2. $\left|Q_{1}, M_{1}\right|:=\operatorname{polar}\left(P_{1}\right)$ and $\left|Q_{2}, M_{2}\right|:=\operatorname{polar}\left(P_{2}\right)$, where $P=\left(\begin{array}{l}P_{1} \\ P_{2}\end{array}\right)$;

3. $F:=\left(\begin{array}{c}-Q_{1} T \\ 0_{n-r \times n}\end{array}\right)$;

4. $\cos \theta:=\left(I+M_{1}\right)^{1 / 2}$ and $\sin \theta:=\left(\left(I+M_{1}\right)^{-1} P_{2}^{t} P_{2}\right)^{1 / 2}$;

5. $G_{+}:=\left[\begin{array}{l}Q_{1} \cos \theta \\ Q_{2} \sin \theta\end{array}\right]$.

In Step 1, the required orthonormal matrix $P$ may be obtained using a $Q R$ factorization of $E$, with column pivoting if we wish $r$ to be minimal and $R(E)=$ $R(P)$.

There are no additional difficulties in computing $G_{-}$and $H_{-}$according to (16)-(17). Only Step 5 of Algorithm 1 needs to be replaced, by

52. $G_{-}:=\left[\begin{array}{c}Q_{1} \sin \theta \\ -Q_{2} \cos \theta\end{array}\right]$.

We give the resulting algorithm the name "Algorithm 1a".

The two polar decompostions and two square roots add to the cost of Algorithm 1. It may well be that the cost of applying $H$ to some large matrix so dominates the cost of contructing it that this is insignificant. We shall try, nevertheless, to reduce this initial cost by seeking alternatives to the representation given by (14)-(15). We therefore seek a new representation of $B$ as $I-G_{+}^{\prime}\left(G_{+}^{t}\right)^{t}$ where $G_{+}^{\prime} \in \mathbf{R}^{m \times r}$. By (20), or equally well by (9) and (13),

$$
\begin{aligned}
\frac{1}{2} S^{t} S & =\frac{1}{2}\left[Q_{1}^{t} Q_{1}+P^{t} P+2 Q_{1}^{t} P_{1}\right] \\
& =I+M_{1} .
\end{aligned}
$$


Now let $R$ be the upper triangular Cholesky factor of $I+M_{1}$. Since the eigenvalues of $I+M_{1}$ are all in $\mid 1,2$, both $R$ and $I+M_{1}$ are extremely well conditioned. Define

$$
G_{+}^{\prime}=\left[\begin{array}{c}
Q_{1} R^{t} \\
P_{2} R^{\cdots 1}
\end{array}\right]
$$

Then

$$
\begin{aligned}
G_{+}^{\prime} & =\left[\begin{array}{c}
Q_{1}\left(I+M_{1}\right) \\
P_{2}
\end{array}\right] R^{-1} \\
& =\left[\begin{array}{c}
Q_{1}+P_{1} \\
P_{2}
\end{array}\right] R^{-1} \\
& =S R^{-1} .
\end{aligned}
$$

Thus, by (21) and (22),

$$
\begin{aligned}
H(S) & =I-S\left(\frac{1}{2} S^{t} S\right)^{-1} S^{t} \\
& =I-S R^{-1} R^{-t} S^{t} \\
& =I-G_{+}^{\prime}\left(G_{+}^{\prime}\right)^{t}
\end{aligned}
$$

Thus we have a second algorithm.

Algorithi 2. Compute $F \in \mathbf{R}^{n \times n}$ and $G_{+}^{\prime} \in \mathbf{R}^{m \times r}, r \leq n$, such that $H E=\bar{F}$, where $H \equiv I-G_{+}^{\prime}\left(G_{+}^{\prime}\right)^{t}$.

1. Find $P \in \mathbf{R}^{m \times r}$ and $T \in \mathbf{R}^{r \times n}$ such that $R(E) \subseteq R(P), P^{\dagger} P=I$, and $P T=E$;

2. $\left|Q_{1}, M_{1}\right|:=\operatorname{polar}\left(P_{1}\right)$ where $P=\left(\begin{array}{l}P_{1} \\ P_{3}\end{array}\right)$;

3. $F:=\left(\begin{array}{c}-Q_{1} T \\ O_{n-8 \times n}\end{array}\right)$;

4. $R:=\operatorname{cholesky}\left(I+M_{1}\right)$;

5. $G_{+}^{\prime}:=\left[\begin{array}{c}Q_{1} R^{t} \\ P_{2} R^{-1}\end{array}\right]$.

Thus we may represent $H_{+}$without having to construct $\sin \theta$ or $\cos \theta$. It is worthwhile asking whether we can do the same for $H_{-}$. We seek $G_{-}^{\prime}$ such that $B_{-}=I-G_{-}^{\prime}\left(G_{-}^{\prime}\right)^{t}$. We may attempt to repeat the derivation above, subetituting $-Q_{1}$ for $Q_{1}$. But there is a cause for concern. The Cholesky factor of $I-M_{1}$ and its inverse may not exist or, worse, may be ill conditioned.

Instead we proceed as follows. Define $P_{-} \equiv \frac{1}{2} G_{-} G_{-}$where $G_{-}$is given by (17). 
Then $H_{-}=I-2 P_{-}$. Furthermore, using (9),

$$
\begin{aligned}
2 P_{-} & =2\left(\begin{array}{c}
Q_{1} \sin \theta \\
-Q_{2} \cos \theta
\end{array}\right)\left(\begin{array}{c}
Q_{1} \sin \theta \\
-Q_{2} \cos \theta
\end{array}\right)^{t} \\
& =\left(\begin{array}{c}
Q_{1} \sin 2 \theta \\
-Q_{2}\left(2 \cos ^{2} \theta\right)
\end{array}\right)\left(2 \cos ^{2} \theta\right)^{-1}\left(\begin{array}{c}
Q_{1} \sin 2 \theta \\
-Q_{2}\left(2 \cos ^{2} \theta\right)
\end{array}\right)^{t} \\
& =\left(\begin{array}{c}
Q_{1} M_{2} R^{-1} \\
-Q_{2} R^{t}
\end{array}\right)\left(\begin{array}{c}
Q_{1} M_{2} R^{-1} \\
-Q_{2} R^{t}
\end{array}\right)^{t} \\
& \equiv G_{-}^{t}\left(G_{-}^{t}\right)^{t} .
\end{aligned}
$$

To summarize, we have

ALGORITHM 3. Compute $F \in \mathbf{R}^{n \times n}$ and $G_{-}^{\prime} \in \mathbf{R}^{n \times r}, r \leq n$, such that $H E=\bar{F}$, where $H \equiv I-G_{-}^{\prime}\left(G_{-}^{\prime}\right)^{t}$.

1. Find $P: \mathbf{R}^{m \times r}$ and $T \in \mathbf{R}^{r \times n}$ such that $R(E) \subseteq R(P), P^{t} P=I$, and $P T=E$;

2. $\left\{Q_{1}, M_{1}\right\}:=\operatorname{polar}\left(P_{1}\right)$ and $\left|Q_{2}, M_{2}\right|:-\operatorname{polar}\left(P_{2}\right)$ where $P=\left(\begin{array}{l}P_{1} \\ P_{2}\end{array}\right)$;

3. $F:=\left(\begin{array}{c}-Q_{1} T \\ O_{n \rightarrow \times n}\end{array}\right)$;

4. $R:=\operatorname{cholesky}\left(I+M_{1}\right)$;

5. $G_{-}^{\prime}:=\left[\begin{array}{c}Q_{1} M_{2} R^{-1} \\ -Q_{2} R^{t}\end{array}\right]$.

Therefore we may again stably construct a representation of $H_{-}$without resort to $\sin \theta$ and $\cos \theta$. But in this case we must compute a polar decomposition (7) of $\boldsymbol{P}_{\mathbf{2}}$.

Thus, the esential difference between the constructions of $G_{ \pm}$and $G_{ \pm}^{\prime}$ is that for $G_{ \pm}$one uses two matrix square roots, to compute $\cos \theta=\frac{1}{\sqrt{2}}\left(I+M_{1}\right)^{1 / 2}$ and $\sin \theta=\frac{1}{\sqrt{2}}\left[\left(I+M_{1}\right)^{-1}\left(I-M_{1}^{2}\right)\right]^{1 / 2} ;$ while for $G_{ \pm}^{\prime}$ one must use a Cholesky factorization of $I+M_{1}$. Furthermore, one may construct $G_{+}$without ever computing the factors $Q_{2}$ and $M_{2}$ of $P_{2}$, which is a distinct advantage.

Still other representations for $\boldsymbol{H}_{ \pm}$are possible. For example,

1. Compute $P_{1}=Q_{1} M_{1}$ and $V=\left(I+M_{1}\right)^{-1}$;

2. $H_{+}=\left(\begin{array}{cc}-P_{1} Q_{1}^{t} & -Q_{1} P_{2}^{t} \\ -P_{2} Q_{1}^{t} & I-P_{2} V P_{2}^{t}\end{array}\right)$.

3.2.1. Efficiency. Algorithms that are couched in terms of modules such as the Basic Linear Algebrta Subprograms [9], matrix-vector products, and matrixmatrix product tend to perform well on modem vector and parallel computers . [5]. In fact, very high speed systolic array devices can be used to implement these operations. One advantage of block reflectors is that they can be computed using matrix multiplication for most of the work, and they can be applied uning matrix 
multiplication for all of the work. Bischof and lan Loan $11_{1}^{1}$ point out that algorithms "rich in matrix multiplication" are attractive for these reasons. Matrix multiply $(n \times n)$ also has the extremely important property that there is substantial reuse of data - $O\left(n^{2}\right)$ data and $O\left(n^{3}\right)$ arithmetic. It is therefore possible to support a processor whose speed is $O(n)$ times greater than the bandwidth of the memory.

In Algorithm 2 above, computation of a block reflector requires

(i) Computation of an orthonormal matrix $P$ such that $E=P T$;

(ii) Polar decomposition of an $n \times n$ matrix;

(iii) Cholesky factorization of an $n \times n$ matrix and inversion of the Cholesky factor;

[iv] Matrix multiplication.

As applied to computation of the block reflector, the operation counts of items [i] and [iv] are $O\left(m n^{2}\right)$ and those of items [ii] and [iii are $O\left(n^{3}\right)$. We are especially interested in the case $m \gg n$.

The computation of $P$ (item [i]) could be done using a $Q R$ factorization (with column pivoting if we wish to make the number of columns of $P$ as small as possible). The implementation suggested by Bischof and Van Loan, which is rich in matrix multiply, could be used. In a later paper, we shall give another algorithm for item [i] that is rich in matrix multiply.

Item [iii] is not matrix multiply. but it is very inexpensive compared to the other items.

The polar decomposition, item |ii;, can also be computed with a procedure dominated by matrix multiply. We start with Higham's method for the polar decomposition of a given nonsingular matrix $A$. In brief, this algorithm constructs a sequence of matrices $\left\{B_{i}\right\}$ where

$$
B_{0}=A
$$

and

$$
B_{i+1}=\frac{1}{2}\left(\gamma_{i} B_{i}+\frac{1}{\gamma_{i}} B_{i}^{-T}\right)
$$

and the scalars $\boldsymbol{\gamma}_{i}$ are chosen by the algorithm to accelerate convergence. The sequence $\left\{B_{i}\right\}$ converges quadratically to the orthogonal polar factor. Higham has shown that 5-6 iterations are typically needed and that the computation time is somewhat less than that for the usual SVD-based method [7].

At each step, $B_{i}^{-1}$ is needed; its computation dominates, only $O\left(n^{2}\right)$ other work is done. For the first step, the inverse can be computed in a conventional way. For all the subsequent iterations of Higham's method, we take advantage of the fact that $B_{i-1}^{-1}$ is a good a priori approximation to $B_{i}^{-1}$, which gets better with incrensing $i$ due to the rapid convergence of $\left\{B_{i}\right\}$. In fact,

$$
\left\|B_{i+1}-B_{i}\right\|=O\left(2^{-z^{i}}\right)
$$


It follows, since $B_{\infty}=Q$ is orthogonal and hence has condition number unity that

$$
\left\|B_{i+1}^{-1}-B_{i}^{-1}\right\|=O\left(2^{-2^{1}}\right) .
$$

Therefore we use Schulz's iterative method $|12|$ as an inner iteration to compute $B_{i}{ }^{-1}$. This matrix iteration produces a sequence $\left\{A_{k}\right\}$ via

$$
A_{k+1}=A_{k}+\left(I-A_{k} B_{\imath}\right) A_{k} . \quad k=0,1, \ldots
$$

where

$$
A_{0}=B_{i-1}^{-1} .
$$

The sequence $\left\{A_{k}\right\}$ converges to $B^{-1}$ quadratically.

Nole that this method is entirely matrix multiply-add. Experiments have shown that five iterations suffice for convergence of Higham's method. The question is, how many iterations of Schulz's method are required. This depends on $i$. Here are typical results:

\begin{tabular}{cc} 
Iteration - & Number of inner iterations $\mathbf{k}$ \\
\hline 1 & 6 \\
2 & 5 \\
3 & 3 \\
4 & 2 \\
5 & 1
\end{tabular}

Thus, about 17 Schulz iterations, or 34 matrix multiplications, are needed for the polar factorization. Were the matrix inverses to be computed directly, five such inverses would have been necessary. Since matrix inversion requires as many floating point operations as matrix multiplication $\left(2 n^{3}\right)$, the matrix multiply oriented version of the algorithm is more efficient if matrix multiply can be done at a rate $34 / 5=$ 6.8 times faster than matrix inversion.

The SVD can also be used to compute a polar factorization. Higham has shown that his algorithm is somewhat less costly, even under the usual model of computational cost.

4. Experiments. Ten very ill-conditioned $12 \times 8$ matrices $E$ were generated by the following procedure. Random matrices $U_{1}$ and $V_{1}$ were chosen and their columns orthogonalized to produce orthogonal matrices $U$ and $V$. Seven random singular values were obtained by sampling the random variable

$$
\sigma:=\epsilon^{1-\mu^{2}}
$$


where $\epsilon=2^{-52}$.was the machine precision and $\mu$ was uniformly distributed in $[0$, 1); the other singular value was taken to be 1 . Finally we computed

$$
E=U \Sigma V^{t}
$$

where $\Sigma=\operatorname{diag}\left(\sigma_{1}, \cdots, \sigma_{8}\right)$. We then computed a $12 \times r$ matrix $P$ with orthonormal columns, and $\operatorname{rank}(E) \leq r \leq 8$. Algorithm 2 was used to find $F$ and $G_{+}^{\prime}$. All computations were performed on an IBM PC/AT using PC MATLAB, which employs IEEE-standard double precision arithmetic, with 15 decimal-digit precision.

Let

$$
H=1-G_{+}^{\prime}\left(G_{+}^{\prime}\right)^{t}=\left[\begin{array}{l}
H_{1} \\
H_{2}
\end{array}\right], \quad H_{1} \in \mathbf{R}^{8 \times 12}
$$

In each case we computed four measures of error.

1. (nonorthogonality): $\left\|I_{12}-H^{t} H\right\|$;

2. (anisometry): $\left\|F^{t} F-E^{t} E\right\|_{\text {; }}$

3. (correctness of $F$ ): $\left\|H_{1} E-F\right\|$;

4. (correctness of $H$ ): $\left\|H_{2} E\right\|$.

The matrix 2-norm was used. The rank of $P$ varied from 3 to 7 . The condition number of $E$ was always at least $2.7 \times 10^{15}$. All the error measures were in the interval $\left[0, .7 \times 10^{-14}\right)$.

Isomorphic experiments were performed using $G_{-}^{\prime}$ (computed using Algorithm 3), $G_{+}$(computed using Algorithm 1), and $G_{-}$(computed using Algorithm 1a), with these results:

0. Errors using $G_{+}^{t}$ were $\leq .7 \times 10^{-14}$;

1. Errors using $G_{-}^{\prime}$ were $\leq .7 \times 10^{-14}$;

2. Errors using $G_{+}$were $\leq .9 \times 10^{-14}$;

3. Errors using $G_{-}$were $\leq 3.0 \times 10^{-14}$.

\section{REFERENCES}

[1] C. BISCHOF AND C. VAN LOAN, The WY representation for products of Householder matrices, SIAM J. Sci. Statist. Comput., 8 (1987), pp. s2-s13.

[2] O. E. BRONLUND AND Th. LUNDE JOHNSEN, QR-factorization of partitioned matrices, Comput. Methods Appl. Mech. Engrz., 3 (1974), pp. 153-172.

(3) C. DAVS and W. M. KAHAN, The rotation of eigenvectors by a perturbation III, this Journal, 7 (1970), pp. 1-16.

(4) G. DIETRICH, A new formulation of the hypermatrix Houscholder-QR decompovition, Comput. Methods Appl. Mech. Engrg., 9 (1976), pp. 273-280. 
[5] J. J. DONGARRA F. G. GuSTAVSON AND A. KARP, Implementing linear algebra algorithms for dense matrices on a rector pipelined machine, SIAM Rev., 26 (1984), pp. 91-112.

[6] G. H. Golub AND C. F. VAN LOAN, Matrix Computations, Johns Hopkins, Baltimore, MD, 1983.

[7] N. J. HIGHAM, Computing the polar decomposition - with applications, SIAM J. Sci. Statist. Compute., 7 (1986), pp. 1160-1174.

[8] L. KAUfMAN, The generalized Householder transformation and sparse matries, Linear Algebra Apple., to appear.

(9) C. L. LAW'SON, R. J. HANSON, F. T. KROGH, and D. R. KINCAID, Basic linear algebra subprograms for FORTRAN usage, ACM Trans. Math. Software, 5 (1979). pp. 308-323.

[10] T. LING, Block householder transformation, Unpublished manuscript, Bathemetics Department, Wuhan University, China, 1980.

[11] B. N. PArtlet, The Symmetric Eigenvalue Problem, Prentice-Hall, Englewood Cliffs, NJ, 1980.

[12] G. SCHuLz, Herative Berechnung der rezipruken Matrix, Z. Angew. Math. Mech., 13 (1933), pp. 57-59.

[13] G. W. STEWART, On the perturbation of pseudo-inverses, projections and linear least squares problems, SIAM Rev., 19 (1977), pp. 634-662.

20 


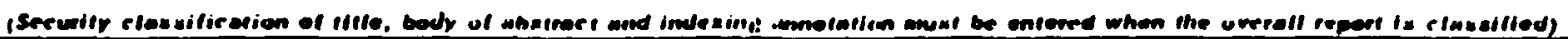
1. Onicina ting ac rivity (Coopunate mother)

Univeratty of California, Berkeley Dncleselfied

J. REROAT TITLE

Block Reflectors: Theory and Computation

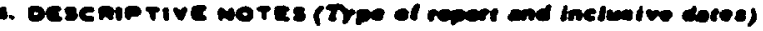

CPal report, Mey 1987

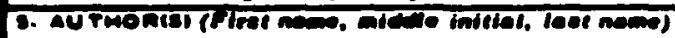

Dobert Schretber and Beresford M. Parlett

\begin{tabular}{|c|c|c|}
\hline $\begin{array}{l}\text { G. ALPOAT OATE } \\
\text { YaY } 1987\end{array}$ & $\begin{array}{l}\text { 70. TOTAL mo. OP Races } \\
20\end{array}$ & 76. wo. of actes \\
\hline $\begin{array}{l}\text { e. } \\
\text { e }\end{array}$ & Wh. opment nctoont molst & 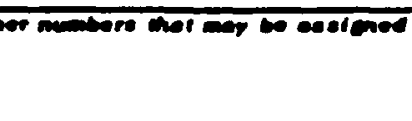 \\
\hline
\end{tabular}

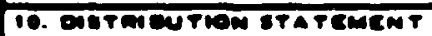

11. Suphomentant worce

12. Soomsonime ontitant activity

Yatheretes Branch

Office of reval Reenrch

inshington, DC 20360

A block reflector is an orthogonal. symmetric mat rix that reverses a suleapace whose dimension may be greater than one. We shall develop the properties of blork refiectors and give some algorithms for computing a block reflector that int rodures a block of zrros into a matrix. Wc consider the compact representation of blosk referturs. some applications, and their use in parallel computers. $(L f)$ 